Relations industrielles

Industrial Relations

Grèves et lock-out au Québec, 1966-1976 : quelques précisions sur les modes de compilation, par François Delorme, Gaspar Lalonde et Lucie Tremblay, Québec, ministère du Travail et de la Main-d'oeuvre du Québec, octobre 1977, 36 pp.

\title{
Jacques Mercier
}

Volume 33, numéro 2, 1978

URI : https://id.erudit.org/iderudit/028882ar

DOI : https://doi.org/10.7202/028882ar

Aller au sommaire du numéro

\section{Éditeur(s)}

Département des relations industrielles de l'Université Laval

\section{ISSN}

0034-379X (imprimé)

1703-8138 (numérique)

Découvrir la revue

Citer ce compte rendu

Mercier, J. (1978). Compte rendu de [Grèves et lock-out au Québec, 1966-1976 : quelques précisions sur les modes de compilation, par François Delorme, Gaspar Lalonde et Lucie Tremblay, Québec, ministère du Travail et de la Main-d'oeuvre du Québec, octobre 1977, 36 pp.] Relations industrielles / Industrial Relations, 33(2), 378-379. https://doi.org/10.7202/028882ar

Tous droits réservés (C) Département des relations industrielles de l'Université Laval, 1978
Ce document est protégé par la loi sur le droit d'auteur. L'utilisation des services d'Érudit (y compris la reproduction) est assujettie à sa politique d'utilisation que vous pouvez consulter en ligne.

https://apropos.erudit.org/fr/usagers/politique-dutilisation/ 
travail à l'université de Bari: l'embauche des travailleurs et les formes progressives de limitation des critères de sélection du personnel, systèmes de classification des travailleurs, «inquadramento unico», professionnalité et organisation du travail, l'ambiance du travail dans les contrats collectifs, certaines considérations sur la négociation des investissements et de la décentralisation de la production et les droits syndicaux ;

- Mario Patrizi, responsable du bureau du développement professionnel et organisationnel de la Société Terni, consacre le dernier chapitre de l'ouvrage à l'exercice de la fonction personnel: fondation, consolidation et recherche d'un nouveau modèle de fonctionnement du système de relations industrielles et exposé de la négociation collective, des nouvelles structures syndicales d'usine, de la formation, de la nouvelle organisation de la direction du personnel, de la recherche sur l'absentéisme et de l'organisation du travail, de 1970 à 1976 à la société Terni.

- Le signataire de ces lignes, seul nonItalien parmi les auteurs de l'ouvrage, se vit confier la rédaction du chapitre sur la communication interne dans l'entreprise: la communication organisationnelle, l' " audit» communicationnel et d'autres développements récents, management et manager de la communication dans l'entreprise (approche multidisciplinaire de la communication, fonction de direction et champ d'études, politique d'information dans l'entreprise vue comme système de communication, la pluralité des sources d'information, les mass media dans l'entreprise, le problème des jeunes, fonction et responsable de l'information), relations publiques et relations industrielles (communication et conflit, les relations publiques internes, les relations humaines et les sciences du comportement, les grèves et l'information interne, avec des références au Canada, une information paritaire).

Il s'agit, dans l'ensemble, d'une œuvre exemplaire, qui n'a pas encore, hélas, son équivalent en langue française. Puissionsnous voir vite nos meilleurs spécialistes québécois, français, belges et suisses, entre autres, remplir un vide et nous donner une vue globale sur une fonction vaste et multiforme en plein développement.
J'ajouterais que ce volume s'inscrit dans une très riche production d'ouvrages en relations industrielles que l'Italie r'a cessé de nous offrir ces dernières années, et qui ne constitue, peut-être, aux yeux du profane, qu'un paradoxe de plus caractérisant un pays pour beaucoup déconcertant mais pourvu d'un potentiel considérable, et qui donne l'impression de se survivre continuellement en attendant d'être gouverné.

\section{Dimitri WEISS}

Université de Paris I Panthéon-Sorbonne

Grèves et lock-out au Québec, 1966-1976: quelques précisions sur les modes de compilation, par François Delorme, Gaspar Lalonde et Lucie Tremblay, Québec, Ministère du Travail et de la Main-d' œuvre du Québec octobre 1977, $36 \mathrm{pp}$.

Étant donné l'objectif sous-jacent dans lequel s'inscrit le présent document, à savoir la prise en charge éventuelle par le ministère du Travail et de la Main-d' ceuvre du Québec du domaine des statistiques sur les arrêts de travail en ce qui concerne le territoire québécois, le lecteur appréciera que l'effort premier des auteurs ait consisté à dégager dans une analyse comparative les problèmes méthodologiques rencontrés dans la production des statistiques sur les arrêts de travail au Canada et au Québec.

L'analyse couvre la définition du terme «arrêt de travail», les sources de données sur le phénomène, la mesure selon le nombre, l'ampleur et la durée des arrêts ainsi que le calcul de la perte de temps de travail qui en résulte. Finalement, diverses données couvrant les périodes 1966-1975 pour les statistiques fédérales et 1976 pour les statistiques provinciales sont reproduites et analysées. Il aurait été intéressant de comparer ces dernières aux statistiques fédérales pour 1976 ce qui aurait permis de mieux distinguer, du moins en partie, les différences entre les deux séries de compilation. Celles-ci sont d'ailleurs multiples; m'entionnons entre autres les sources de cueillettes de données, la période de référence, le calcul des jours-hommes perdus, du pourcentage du temps ouvrable, la me- 
sure des conflits, etc. Ce qui nous amène à se demander si l'on ne va pas tout simplement se retrouver dorénavant avec deux séries portant sur la mesure du même phénomène mais qu'on ne pourra pas comparer.

Enfin si telle n'est pas leur préoccupation première, les auteurs ne manquent jamais l'occasion de faire les mises-engarde qui s'imposent au niveau de l'utilisation et l'interprétation de ces données dans l'analyse de problème divers. Ce point contribue davantage à faire de ce document un outil méthodologique fort précieux.

Jacques MERCIER

\section{Université Laval}

Out of the Sweatshop: The Struggle for Industrial Democracy, Leon Stein (ed.), New York, The New York Times Book, Co., 1977, 367 pp.

Cet ouvrage relate, à travers près de 170 textes, la difficile implantation du syndicalisme dans l'industrie du vêtement aux États-Unis. Le «combat pour la démocratie industrielle» que Leon Stein veut décrire est celui de l'International Ladies' Garment Workers' Union (ILGWU). Stein caractérise les principales étapes de l'évolution de l'ILGWU à l'aide de textes extraits d'articles de journaux, de rapports de commissions d'enquête, d'ouvrages historiques, de discours politiques, de romans, etc. Les textes sont regroupés en chapitres qui traitent d'événements ou de séquences d'événements spécifiques ou encore de thèmes généraux comme ceux de la difficulté de normaliser les salaires et les exigences de la production dans l'industrie du vêtement (chap. 12, "Pay and Production») et des problèmes de la syndicalisation des employés des entreprises très mobiles de ce genre d'industrie (chap. 13, «Old Battles, New Frontiers»). De nombreuses photographies et illustrations accentuent le réalisme de la description qui est faite des conditions de travail pénibles du secteur du vêtement.

Le «sweating system», dont la pratique est loin d'être disparue, s'est développé, aux États-Unis, grâce à une maind'œuvre docile et particulièrement vulnérable: celle des immigrants qui avaient fui des régimes autoritaires, comme celui de la Russie tsariste et qui étaient venus chercher en Amérique une liberté et une prospérité qui s'avèrent rapidement illusoires (chap. 2, "Sweatshop: Immigrant Style»). Un témoin de l'époque, Florence Kelley - qui fut le premier inspecteur d'usine de l'Etat d'Illinois - rapporte, en 1899 , qu'il n'y a à peu près pas d'américains d'origine dans le secteur du vêtement: "we never found any in sweatshops. The Native Americans who are working at the garment trades are perhaps the most dangerous of all from the point of view of the purchaser» (pp. 32-33). Il va sans dire que ce milieu n'était pas particulièrement perméable au syndicalisme (chap. 3, «Years of Survival»): "The Labor force consisted of ethnic and national minorities, immigrants who were either too beaten or too ambitious to support a union. They were trapped by a mode of production that camouflaged the employer and defied their efforts to locate the center of industrial responsibility» (p. 40).

Ce n'est qu'après plusieurs tentatives inefficaces que l'ILGWU est formée en 1900 ; elle connaît sa première grève importante - qui dure 2 mois - en 1909, (chap. 4, "The Shirtwaist Makers' Strike, $1909 »)$. Elle est suivie d'une autre grève déterminante qui se règle par la conclusion d'un accord collectif s'appliquant à l'ensemble de l'industrie (chap. 5, "The Cloakmakers' Strike, 1910 » et chap. 6 "The Protocol of Peace: its Rise»). Cet accord assurait l'existence de l'ILGWU et précisait le champ du négociable: échelle de salaires, modes d'arbitrage des conflits, conditions minimales de travail, etc. L'accord sera abrogé en 1916. (chap. 7, «The Protocol of Peace; Its Decline»). Il aura été l'occasion pour le syndicat de déborder les revendications strictement salariales et de se sensibiliser à des questions comme celles de la santé, de l'éducation et de l'action politique (chap. 8, "Life and Death» et chap. 11, «Way of Life»).

L'ILGWU subit les contrecoups de la crise économique pendant les années 1930; elle revit avec le «New Deal» du président Roosevelt qui entraine de plus grandes possibilités d'organisation syndicale (chap. 9, «Rebirth of the Union» et chap. 10 «The New Deal»). Depuis l'ILGWU est devenu un syndicat puis- 